\title{
Proximal Phalanx of Foot
}

National Cancer Institute

\section{Source}

National Cancer Institute. Proximal Phalanx of Foot. NCI Thesaurus. Code C52785.

One of five small bones located in the foot, which form the base of each toe, and which articulates proximally with the head of a metatarsal bone. 